\title{
A Comparative Analysis of Local and Global Adaptive Threshold Estimation Techniques for Energy Detection In Cognitive Radio
}

\author{
A. J. Onumanyi ${ }^{1, *}$, A. M. Abu-Mahfouz ${ }^{1,2}$, G. P. Hancke ${ }^{3,1}$ \\ ${ }^{1}$ Department of Electrical, Electronic and Computer Engineering, University of Pretoria, South Africa \\ ${ }^{2}$ Meraka Institute, Council for Scientific and Industrial Research, Pretoria, South Africa \\ ${ }^{3}$ Department of Computer Science, City University of Hong Kong, Hong Kong, China \\ *Corresponding Author: adeiza1@ yahoo.com
}

\begin{abstract}
In this paper, we compare local and global adaptive threshold estimation techniques for energy detection in Cognitive Radio (CR). By this comparison, a sum-up synopsis is provided regarding the effective performance range and the operating conditions under which both classes best apply in CR. Representative methods from both classes were implemented and trained using synthesized signals to fine tune each algorithm's parameter values. Further tests were conducted using real-life signals acquired via a spectrum survey exercise and results were analysed using the probability of detection and the probability of false alarm computed for each algorithm. It is observed that while local based methods may be adept at maintaining a low constant probability of false alarm, they however suffer a grossly low probability of detection over a wide variety of CR spectra. Consequently, we concluded that global adaptive threshold estimation techniques are more suitable for signal detection in CR than their local adaptive thresholding counterparts.
\end{abstract}

Keywords

Cognitive Radio, Comparative Analysis, Energy Detector, Global, Local, Threshold

\section{INTRODUCTION}

Cognitive Radios are smart radios designed for dynamic and opportunistic communication in which channels are utilized if detected to be free (signal absent), and vacated if occupied (signal present) [1]. CRs are now widely considered in the wake of the spectrum scarcity/underutilization problem currently beleaguering the wireless communication industry. However, the potency of CR systems greatly depends on how well existing spectrum sensing (SS) methods are improved upon [2]. In this regard, one popular method widely used for spectrum sensing is the Energy Detection (ED) approach known for its quick sensing capability, simple design and its independence of the Primary User (PU) signal waveform [1], [3], [4]. Essentially, the ED approach determines if a band is free (noise only) or not by comparing the energy level in a channel to an estimated threshold value. It is expected that the estimated threshold value should be above the noise level in the channel. However, in situations where the noise estimate is unknown a priori and unstable, adaptive threshold estimation techniques become essential to guarantee minimal error rates in the CR decision making process.

Adaptive Thresholding Techniques (ATTs) are defined in CR as methods deployed for estimating the threshold of an ED without prior knowledge of the noise power in the measured spectra [1]. They are particularly important for improving the performance of the ED in vacillating noise floor conditions where the effect of noise uncertainty is known to be significant [5]. It is perhaps obvious that without the use of ATTs, the ED performs poorly in noise uncertainty regimes resulting in increased false alarm (spectra underutilization), and missed detection rates (signal interference) [6], [7]. For these reasons, ATTs have become fundamental components in most modern EDs, thus advancing their use in recent industrial applications such as in cyber physical systems (CPS), low power wide area networks (LPWANs), smart grids [8], industrial wireless sensor networks [9], [10], and in smart metering, and smart city applications [11].

We classify ATTs into two basic types namely the Local and the Global based methods. We define the local thresholding methods in CR as methods that compute a different threshold value for each sample in the measured spectra. These are elsewhere referred to as constant false alarm rate (CFAR) detectors [7], [12]-[14]. On the other hand, we define global thresholding methods as methods that compute a single threshold value over an entire range of samples in a reference window (or measured spectra). We liken this to the global thresholding concept in Image Processing. An overview of both classes is presented in Section 2 including notable examples.

The literature teems with different ATTs belonging to both classes, however, an obvious lack of a systematic comparative analysis of both classes is observed. This lack limits the ability of the CR Engineer to clearly state with certainty which class 
best suits the threshold estimation process in CR. Consequently, CR Engineers may find it difficult choosing a specific ATT class for application in new CR based programs. Furthermore, it may be essential to determine the operating conditions under which both classes perform well. Thus, in this paper, we describe a general methodology for comparing selected techniques from both classes based on a common set of input signals, and on some known performance metrics for the purpose of analysis. Synthesized dataset were used in Monte Carlo simulations to train the different ATTs considered in this paper. This was done to set the different parameter values of each algorithm (see discussion in Section 3). Real-life dataset were used for evaluation and can be freely accessed in [15] for further research. The results of our analysis and further discussions are presented in Section 4, while conclusions are drawn in Section 5.

\section{An OVERView of RELEVANT Literature}

In this section, we overview a few of the existing ATTs adopted for SS in CR. These methods are classified and discussed according to the two groups namely the Local and the Global based methods.

\subsection{Local Thresholding Techniques}

We define local thresholding techniques (LTTs) as methods that estimate a unique detection threshold per sample in a reference window (or measured spectra) as illustrated in Fig. 1a. The concept of local thresholding in CR can be likened to that found in Image Processing. Essentially, LTTs work by varying the threshold value computed per sample in a measured band towards maintaining a constant false alarm rate (see the varying threshold values in Fig. 1a).

An example of a popular LTT that quickly comes to mind is the cell averaging CFAR (CA-CFAR) [16]. This technique declares that a target (or Primary User (PU)) signal is present if the power of the sample in the cell under test (CUT) is greater than the power of the samples in its adjacent cells and the local average power level. It is a simple approach in which the estimate of the local power level corresponding to the noise power may be increased to cater for the case of possible limited sample sizes. Soon after the CA-CFAR processor was proposed, several variants rapidly appeared to include the "greatest of" (GO-CFAR) [17], the "smallest of" (SO-CFAR) [18], the heterogeneous clutter estimating (HCE-CFAR) [19], the 'trimmed mean' (TM-CFAR) [12], and the ordered statistics (OS-CFAR) [20]. Other known methods include the automatic censored mean level detector (ACMLD-CFAR) [21], the generalized two level censored mean detector (GTLCMLD-CFAR) [21], and the variability index CFAR (VI-CFAR) [22]. These methods address issues pertaining to improving either the detection or the false alarm rate particularly in non-homogeneous clutter regimes.

Other variants of the CA-CFAR processor are noted to include the maximum likelihood and algebraic product (MLAP-CFAR) [23], and the goodness-of-fit and generalized likelihood test with dual censoring (GGDC-CFAR) [24]. Essentially, these LTTs were originally conceived for Radar detection problems but have found widespread application in CR. For example, the CACFAR method was adapted for use in CR in [7], [13], [14]. The results obtained show that these methods possess the potential to improve the performance of the ED in CR. Thus, it is considered worthwhile to investigate and examine representative methods in this class based on a common comparative platform.

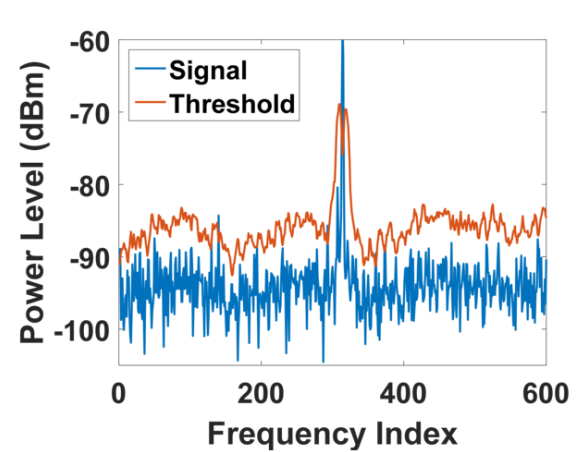

(a)

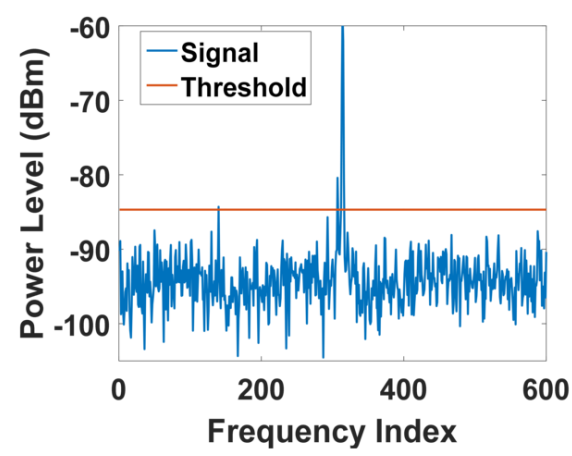

(b)

Fig. 1: Two different approaches to Adaptive Threshold Estimation: (a) LTT and (b) GTT 


\subsection{Global Thresholding Techniques}

Global thresholding techniques (GTTs) are defined as methods that compute a single threshold value over a contiguous number of samples in a specified window (or measured spectra). An example of a single threshold value computed using a GTT is shown in Fig. 1b. This illustration differentiates the threshold process of the GTT from the LTT. Thresholds computed by GTTs are different from the concept of fixed thresholding. In fixed thresholding, a preestablished threshold value never changes even when the dataset changes, however, in GTT, a new threshold value is continuously computed for each newly measured spectra.

There are quite a number of GTTs used in the ED for CR. An early GTT was developed by Lehtomaki et al., [7], [13] in which they advanced the use of two different forward methods for the adaptive threshold case. Their methods involved reordering the measured sample sets, and computing the mean of the smallest sample values in an initial window (see [7]). Though simple and adaptive, their approach requires the fine tuning of a few parameters for performance regulation. In further works, Lehtomaki et al., in [14], [25] developed the Forward Consecutive Mean Excision (FCME) algorithm into a variant of other techniques such as the localization algorithm based on double-thresholding (LAD) with the adjacent cluster combining (ACC), the Median FCME (MED-FCME), and the $m$ - $d B$ method based on the MED-FCME. These methods belong to the GTT class and their application to CR is well documented [14], [25].

Other known GTTs can be found in [26]-[32]. These methods are typically characterized by parameters that must be fine tuned towards improving the detector's performance. A few classical GTTs that depend on less than two tunable parameters include the Recursive One-sided Hypothesis Testing (ROHT) algorithm [6], [33], and the First Order Statistical Technique [34]. Other parameterized GTTs belonging to the double threshold class can be found in [35]-[41]. They typically depend on a pair of well chosen fixed threshold values that are invariant to the changing noise level. These methods require a careful choice of the fixed threshold values to guarantee improved performance particularly when sensing conditions abruptly change. The GTT method of Martinez et al., [42] depends on a correlation function to improve the accuracy of noise estimation, while the method of Liu et al., [43] does not require the noise power nor a threshold to be known apriori. Some other methods belonging to the GTT class were found in [26], which was based on the expectation maximization algorithm, and in [44], based on compressive sensing. Furthermore, a few fully automated GTTs were published by Datla et al., in [6], [33] based on the use of Otsu's algorithm [45]. These algorithms [6], [33] are void of tunable parameters, and thus considered fully autonomous. Recently, the method in [6], [33] was modified and improved for CR in [46] to solve problems associated with gray level computations, unimodal histogram properties, and vector dataset thresholding. Essentially, the methods in [6], [33], [46] are a unique class of autonomous GTTs.

From the foregoing, it is quickly obvious that a number of GTTs exist for SS in CR. These methods form a large percentage of the known ATTs available for threshold computation in CR. Nevertheless, it is noteworthy that the performance of the GTTs in comparison to LTTs in CR remains an unexplored exercise. Thus, this lack limits our knowledge regarding which class best suits the threshold estimation process in CR. It may be further essential to establish the conditions under which each class outperforms the other, thus providing numerical evidences to enhance the CR engineer's choice of a suitable method for SS in CR. These are conditions that fully motivate the purpose of this paper.

\section{Methodology}

Our methodology followed the process depicted in Fig. 2. The input signals were acquired, the respective ATTs were implemented, and performance analysis was conducted.

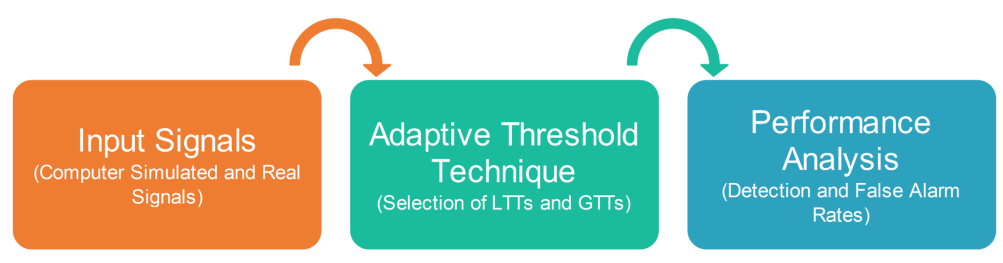

Fig. 2: Processes in our Research Study

\subsection{Input Signals}

Two types of input signal sets (dataset) were used in our investigation. These were the computer simulated and the real-life dataset. The computer simulated dataset were used to train the respective ATTs, while real-life signals were used to test the different algorithms. Noting that CRs are intended for use in the TV band [47], we focused on signals that exist in these 
TABLE I: Parameters used for simulating the OFDM signal

\begin{tabular}{cc}
\hline Parameters & Values \\
\hline Number of carriers $K$ & 1705 \\
Value of carrier number, $K_{\min }$ & 0 \\
Value of carrier number, $K_{\max }$ & 1704 \\
Duration, $T_{U}$ & $224 \mu \mathrm{s}$ \\
Duration of guard band, $\Delta$ & $56 \mu \mathrm{s}$ \\
\hline
\end{tabular}

bands. Consequently, we considered the following input signals: Frequency Modulated (FM), Analog TV (ATV) and Digital TV (DTV) signals. For the DTV case, Orthogonal Frequency Division Multiplexing (OFDM) signals were used. The training phase enabled us to determine the global parameter values of the each algorithm considered in our experiments.

Computer Simulated Datasets: The basic functions governing the computer simulated dataset used in our work are presented. Only Frequency domain signals were used as inputs into the respective ATTs. This ensured that free bands were easily identified by reading off the frequency values along the abscissa of the detector calibrated in Hertz. Once encoded, data generation and the subsequent training processes were conducted via Monte Carlo simulations. For brevity, the FM signal function used and its respective parameter definitions are given:

$$
S_{F M}(k)=\left|\sum_{n=0}^{N-1}\left(A_{c} \cdot \cos \left[2 \pi f_{c} n T+k_{f} \cos \left(2 \pi f_{m} n T\right)\right]\right) e^{-j \omega_{k} n}\right|^{2}, \quad k=0,1, \ldots, N-1
$$

where $S_{F M}(k)$ denotes the spectra of the FM signal for different frequency indices $k=1,2, . ., N-1$, parameters $A_{c}$ and $f_{c}$ denote the amplitude and center frequency of the carrier signal, respectively, $f_{m}$ is the frequency of the modulating signal (baseband), $k_{f}$ the modulating index, $N$ is the total number of frequency samples, $n$ is the index of the time domain signal, and $T$ is the sampling period. The real components of the signals were considered accounting for the square law operation used in (1).

For the DTV case, we considered the 2k mode of the Digital Video Broadcasting Terrestrial (DVB-T) standard, which is based on the OFDM signal. The function used is given as

$$
\begin{aligned}
s(t) & =\operatorname{Re}\left\{e^{j 2 \pi f_{c} t} \sum_{k=K_{\min }}^{K_{\max }} C_{0,0, k} \cdot e^{j 2 \pi k^{\prime}(t-\Delta) / T_{U}}\right\} \\
\text { with } k^{\prime} & =k-\frac{\left(K_{\max }+K_{\min }\right)}{2}
\end{aligned}
$$

where $s(t)$ denotes the OFDM symbol, $f_{c}$ is the central frequency of the radio frequency signal, $k^{\prime}$ is the carrier index relative to the center frequency, $k$ denotes the carrier number, $K_{\max }$ and $K_{\min }$ are the minimum and the maximum number of carriers, $C_{0,0, k}$ is the complex symbol for carrier $k$ of the first Data symbol in the first frame, $T_{U}$ is the inverse of the carrier spacing, and $\Delta$ is the duration of the guard interval. The frequency response of (2) for a 4-QAM symbol dataset was then computed using the Fast Fourier Transform (FFT) algorithm, which was similarly used to compute (1). Essentially, both signal types in (1) and (2) were simulated using the parameter values given in Table I. The simulated signal sets based on eqns (1) and (2) were corrupted using randomly generated Additive White Gaussian Noise (AWGN) to model the impact of channel conditions on the received signal.

Noise only dataset were used to set and validate the parameter values of the different algorithms. Noise only spectra were simulated for a thermal noise power of $-106 \mathrm{dBm}$ calculated for a bandwidth of $6 \mathrm{MHz}$ (for typical TV bands), at a room temperature of $290^{\circ} \mathrm{K}$, using the Boltzmann's constant. The average noise power was increased in steps of $1 \mathrm{~dB}$ to simulate different noise uncertainty levels. This was achieved using a uniform distribution model designed with a $1 \mathrm{~dB}$ variance on the random open interval $(0,1)$.

Real-life Datasets: A spectrum survey exercise was conducted to acquire real-life signals for evaluating the different ATTs examined in this work. The exercise involved the use of a Rigol DSA1030 spectrum analyzer with a frequency range from $9 \mathrm{kHz}$ to $3 \mathrm{GHz}$. The analyzer has a resolution bandwidth (RBW) range of $100 \mathrm{~Hz}$ to $1 \mathrm{MHz}$, in 1-3-10 sequence, and a video bandwidth (VBW) range of $1 \mathrm{~Hz}$ to $3 \mathrm{MHz}$. Using a RBW of $100 \mathrm{~Hz}$, a VBW of $10 \mathrm{~Hz}$, an average detector, and a trace average $\geq 50$, we began our experiments with a measured displayed average noise level of $-135 \mathrm{dBm} / \mathrm{Hz}$. In our experiments, the Preamplifier of the analyzer was kept on, using an average RMS trace detector and a dBm scale unit. The 
input of the analyzer was coupled to a VHF/UHF broadband TV Log Periodic antenna having an inbuilt low noise amplifier. Specifically, the SAS-510-2 Log Periodic antenna of A.H. Systems Incorporated was used in our work. The antenna provided an input frequency range from $290 \mathrm{MHz}$ to $2 \mathrm{GHz}$, covering the entire VHF/UHF band being considered for TV whitespace deployment in CR. In addition, the antenna provided an antenna factor of $14-32 \mathrm{~dB}$ and a Gain of $6.5 \mathrm{dBi}$ (specified in the manufacturer's datasheet). This antenna was considered for its excellent cross-polarization property, which greatly reduced the level of measurement uncertainty. Because it is constructed from lightweight aluminium and also directional, it was found suitable for our data acquisition exercise to ensure that maximum signal gain was achieved. A schematic of the experimental setup is shown in Fig. 3. Summarily, a resolution/video bandwidth of $100 \mathrm{~Hz}$ was used for the Rigol spectrum analyzer, with a sweep time range of $300-900$ seconds per band. An average detector was used with an attenuation of $0 \mathrm{~dB}$ over a single sweep mode. The data acquisition sites used were obstruction free to enable clear reception of real-life signals. Because our measurement campaign/setup was situated in the open field to prevent signal obstruction at an altitude of $250 \mathrm{~m}$ above sea level, a power inverter was deployed during the measurement campaign to guarantee continuous power supply whenever the analyzer's inbuilt battery expired. The signal values acquired during our campaign were Power spectra Density (PSD) measurements expressed in $\mathrm{dBm} / \mathrm{Hz}$. For every spectra sweep of the analyzer, the PSD dataset were obtained in Comma-seperated value (CSV) file format and each file was saved in a general purpose laptop for onward analysis.

A summary of the different real-life dataset obtained and the related CR conditions described by each dataset is discussed. The spectra of each signal type considered here can be seen in the results section. The FM broadcast band between 88 $108 \mathrm{MHz}$ was swept to acquire signals revealing quite a highly occupied band. The signal of a specific FM radio station was captured at $90.5 \mathrm{MHz}$ as a means to examine narrowband sensed signals. An Analog TV signal was captured at channel 7, while a Digital TV (DTV) spectra was acquired via channel 56. The DTV spectra represents a real-life OFDM signal essential for examining the different ATTs. The case of differences in the dynamic range of signals was examined by capturing two different closely spaced FM radio signals, while a sparse wideband sensed signal was also acquired to examine the different algorithms. The respective ATTs were also examined based on a real-life noise only spectra measurement. The entire real-life dataset used in this work can be freely accessed by other researchers in [15].

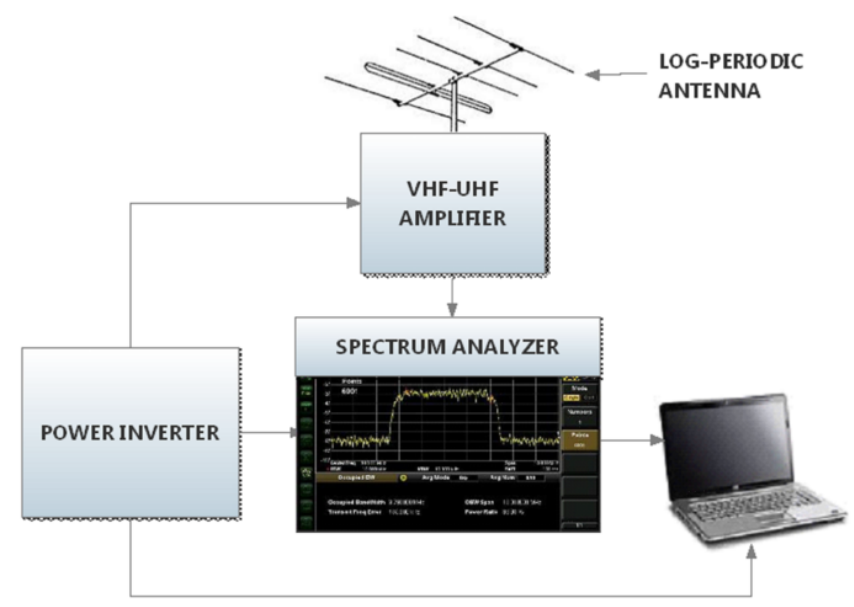

Fig. 3: Experimental Setup for Spectrum Survey Exercise

\subsection{Choice of the respective Adaptive Thresholding Techniques}

Different ATTs were chosen from both classes for evaluation in this work. For the class of LTTs, mainly foundational techniques were considered as they form the basis against which most techniques are compared with in the literature [24]. Thus, the following methods were chosen from the class of LTTs: the Cell Averaging CFAR (CA-CFAR) [16], the Smallest Of Cell Averaging CFAR (SOCA-CFAR) [48], and the Greatest Of Cell Averaging CFAR (GOCA-CFAR) techniques [17]. For the choice of representative methods belonging to the class of GTTs, techniques having two or less than two parameters for controlling the performance of the algorithm were considered for sake of simplicity. In addition, techniques considered to be fully autonomous, which implies being parameterless were also considered. These characteristics are fundamental requirements for maintaining the simplicity, speed and efficiency of the ED for SS in CR [46]. Thus, for these reasons, the following algorithms were considered for the class of GTTs: the Recursive One-sided Hypothesis Testing (ROHT) [6], the First Order Statistical Technique (FOST) [34], the Forward Consecutive Mean Excision (FCME) [7], the Otsu's method (OM) [6], and the Modified Otsu's algorithm (MOA) [46]. These techniques were chosen only as representatives of the different classes. Essentially, our 


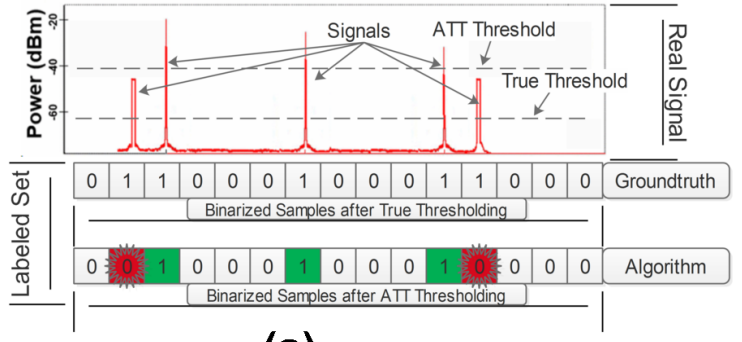

(a)

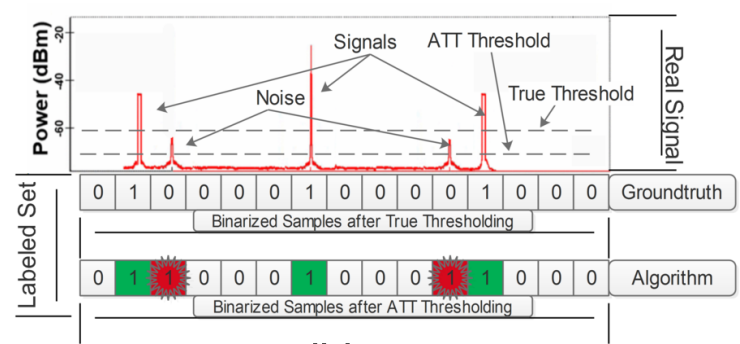

(b)

Fig. 4: Method of Labeling and Analysis showing different errors: (a) Missed Detection, and (c) False alarms

research focused on examining the two classes in a broad sense, and not necessarily on the individual algorithms. For space, we do not describe each algorithm, but rather direct interested readers to the respective references associated with each algorithm. Each algorithm was encoded according to the pseudocode and description given by their respective authors.

\subsection{Method of Performance Analysis}

We analyzed and compared both classes of ATTs using the probability of detection, $P_{D}$, and the false alarm probability, $P_{F A}$ statistically described as follows:

$$
\begin{aligned}
P_{D}=\operatorname{Pr}\left(S(k)>\gamma \mid H_{1}\right), & k=1,2, \ldots, N \\
P_{F A}=\operatorname{Pr}\left(S(k)>\gamma \mid H_{0}\right), & k=1,2, \ldots, N
\end{aligned}
$$

where $S(k)$ denotes the signal spectra values for different frequency indices, $k=1,2, \ldots, N, \gamma$ is the threshold value typically estimated by the respective ATTs, $H_{0}$ is the null hypothesis describing the noise only spectra, and $H_{1}$ is the alternate hypothesis describing the signal plus noise spectra. We computed (4) and (5) following Fawcett's empirical approach [49].

An illustration of our approach is presented in Fig. 4. To describe our approach, it is noted that the measured received signal is all that any CR enabled device may have access to towards making decision about the presence/absence of PU signals in a specific band. Consequently, in our experiments, two factors where known apriori to justify our approach of analysis, namely:

1) The noise level, both of the device and of the ambient values, were measured and thus accounted for apriori to serve as the true threshold value for the groundtruth dataset used in our experiments.

2) The bands occupied by the PU signals in our local environment were also known prior to the measurement campaign following the chart provided by National frequency policy makers in the frequency allocation table.

Having considered these factors, it became quite dependable and reliable to develop the groundtruth used for analysis and comparison in our work. Nevertheless, it is noted that stray signals found using the analyzer but not legitimately assigned within the frequency allocation table in our local environment were not considered as PU signals during our analysis. This prior knowledge ensured that only the correct locations of PU signals in our environment were considered in our analysis. Consequently, the individual threshold-setting methods were then equally and fairly compared against the same measured dataset to provide meaningful results.

Essentially, each dataset (either simulated or real-life) was labeled via a binary approach as the groundtruth using the apriori known true threshold value (see Fig. 4). To determine this true threshold value for the acquired real-life signals, we estimated the analyzer's noise floor by using a $50 \mathrm{ohm}$ resistor connected to the analyzer's input. This ensured that only the noise floor of the analyzer was known at the time. However, this did not guarantee accurate knowledge of the ambient noise level at the time upon connecting the antenna, because the ambient noise values were received along with the actual PU received signal. Consequently, the final true threshold was estimated after true knowledge of the device noise floor along with an addition of a maximum $1 \mathrm{~dB}$ allowance to cater for possible ambient noise uncertainty levels. This approach popularly referred to as the "m-dB approach" has been used extensively in the literature [25], [50], [51]. Thus, following this approach, it was ensured that only about $1 \mathrm{~dB}$ may have been lost during the SNR measurement process, particularly for PU signals that may have been buried in noise at that level. In this case, samples above the true threshold are true signals and labeled as ones, while samples below the true threshold are noise samples and thus labeled as zeros. This labeling process for the groundtruth was carried out for all dataset used in this work. Thereafter, each dataset was relabeled in a binary fashion based on the threshold values computed by the respective ATTs. Essentially, a missed detection error is declared if the labeled groundtruth is 1 and the ATT labeled dataset is 0 (see Fig. 4a), while a false alarm is declared if the labeled groundtruth is 0 and the ATT labeled dataset is 1 (false positive (see Fig. 4b)). Furthermore, a true detection is declared when values in the both the groundtruth and the ATT 
TABLE II: Parameter Settings for the different ATTs

\begin{tabular}{|c|c|c|c|c|c|c|c|}
\hline Class of ATT & Algorithms & Threshold Factor & $P_{F A}(\%)$ & Training Cells & Guard Cells & Initial Clean Set (\%) & Error Limit \\
\hline \multirow{3}{*}{ GTTs } & ROHT [6] & 1.6 & 6.8 & - & - & - & 0.9 \\
\hline & FOST [34] & 1.6 & 6.8 & - & - & - & - \\
\hline & FCME [7] & 1.8 & 6.8 & - & - & 10 & - \\
\hline \multirow{3}{*}{ LTTs } & CA-CFAR [16] & 1.6 & 6.4 & 32 & 2 & - & - \\
\hline & SOCA-CFAR [48] & 1.5 & 6.8 & 32 & 2 & - & - \\
\hline & GOCA-CFAR [17] & 1.65 & 6.4 & 32 & 2 & - & \\
\hline
\end{tabular}

labeled dataset are 1 (true positive). Consequently, by using the knowledge of the confusion matrix and Fawcett's algorithm in [49], the probability of detection per dataset was computed using

$$
P_{D}=\frac{\phi}{P},
$$

where $\phi$ denotes the total number of true positives (truly detected signal samples) if $Y(k)>\gamma \mid H_{1}$, and $P$ is the total number of actual true signal samples (obtained as the total number of ones in the groundtruth). The false alarm probability per dataset was computed using

$$
P_{F A}=\frac{\varphi}{N},
$$

where $\varphi$ denotes the total number of false positives (falsely detected signal samples) if $Y(k)>\gamma \mid H_{0}$, and $N$ is the total number of noise samples (obtained as the total number of zeroes in the groundtruth). In addition, rather than employing the well known Receiver Operating Characteristic (ROC) curve (that is, $P_{D}$ vs $P_{F A}$ ) as in [49], we generated the curves of the probability of false alarm and detection against respective thresholds, that is $P_{D}, P_{F A}$ vs $\gamma$ as a means to easily extract the corresponding point performance of each algorithm (as tabulated in the result section).

\section{RESUlts AND Discussion}

The results are presented in three folds with different interests in mind. First, the spectra is presented showing the different thresholds computed by the different algorithms from both classes. The probability of detection and the probability of false alarm corresponding to each spectra is presented, from which the individual point performances of the different ATTs are obtained and tabulated. Each spectra describes a different condition under which each algorithm may operate. The results are categorized according to the training and the testing phase of our experiments. With regards to the graphical presentation of our results, it is worth noting that each third subfigure showing the probability of detection and the probability of false alarm was plotted for each real-life dataset under consideration at the time. Each performance plot was generated using the true groundtruth of the particular dataset under consideration at the time. Consequently, the point performances of each technique were read-off the respective third subfigure plots using the single threshold value estimated by each technique. These point performances along with the respective threshold values estimated by each technique are then reported in respective performance tables.

\subsection{Training Phase}

In the training phase, the different parameter values belonging to the different respective ATTs were determined. For fairness, the same synthesized dataset were used to train each algorithm. The FM, DTV and noise only dataset were used (refer to Section 3.1). The IEEE 802.22 standard specification guided the bounds that were used to fine tune the parameters of each algorithm. These specifications state that the limits for the probability of detection should be greater than $90 \%$, while simultaneously maintaining a false alarm rate below $10 \%$ [47]. Consequently, we chose to maintain a false alarm rate of $\approx 6.4$ to $6.8 \%$ in the synthesized noise only dataset, and a detection rate of $\approx 95 \%$ in the signal plus noise dataset. A Monte Carlo simulation over a 1000 trials was adopted. The parameter settings of each algorithm from the training phase is presented in Table II. Each algorithm is related to its own parameter setting as can be seen in Table II. For example, the algorithms belonging to the GTT class do not require that a number of training and guard cells be preset, rather, the ROHT requires an error limit to be set as 0.9 (see Table II). Likewise, the FCME requires an initial clean set of $10 \%$ to be preset. However, all the algorithms considered in our evaluation required a threshold factor to be preset as indicated in Table II. These values were subsequently unchanged during the test phase as this may be expected under real-time deployments.

\subsection{Test Phase}

Typical CR devices may need to operate within different communication bands, thus detailed examinations were conducted in this regard and the results obtained are reported. We categorize our results according to the different types of signals examined. 
TABLE III: Detection and False Alarm Rate Performance for the different ATTs for the Real Noise dataset (True Threshold $=-73 \mathrm{dBm}$

\begin{tabular}{cccc}
\hline Class of ATT & Algorithms & Threshold (dBm) & $P_{F A}(\%)$ \\
\hline \multirow{4}{*}{ GTTs } & MOA [46] & -77.00 & 55 \\
& Otsu [6] & -77.06 & 55 \\
& ROHT [6] & -75.34 & 5 \\
& FOST [34] & -75.34 & 5 \\
& FCME [7] & -74.14 & 0 \\
\hline \multirow{3}{*}{ LTTs } & CA-CFAR [16] & - & 0.3 \\
& SOCA-CFAR [48] & - & 4.2 \\
& GOCA-CFAR [17] & - & 0.2 \\
\hline
\end{tabular}

4.2.1 Real-life Noise Only $(132-133 \mathrm{MHz})$ : The spectra of real-life noise samples were used to evaluate the performance of the different algorithms. These samples were collected within the band of $132-133 \mathrm{MHz}$ over the spectrum survey exercise. The respective estimated threshold values computed by each algorithm are depicted in Fig. 5a (for the GTT class) and in Fig. 5b (for the LTT class). The Receiver Operating Characteristic (ROC) curve for the real-life noise dataset is provided in Fig. 5c. The true threshold used to construct the groundtruth for this dataset was known apriori at $-73 \mathrm{dBm}$. The point performance values corresponding to each algorithm were determined from this graph (Fig. 5c), and presented in Table III. Noting that only noise samples were considered in this case, it is obvious that only the probability of false alarm, $P_{F A}$, was computed. It is also noteworthy that the LTTs typically compute a set of threshold values wherein each indexed threshold value corresponds to a similarly indexed sample in the dataset. Thus, unlike the GTTs, no single threshold value can be stated for the LTTs (this can be seen generally in the tabulated results). From the results obtained in this case, it is seen that the LTT class all maintained very low false alarm rates, particularly well below the set value of $\approx 6.8 \%$. In the GTT class, only the Modified Otsu Algorithm (MOA) and the Otsu algorithm failed to maintain a predefined false alarm rate (as seen in Table III. Essentially, unlike the other algorithms (including the LTT class) considered here, both the MOA and the Otsu algorithm belong to the autonomous category. This implies that these algorithms were not predefined to any false alarm rate because they are parameter-less algorithms. Thus, their poor performance in the noise only case can be traced to the presence of a unimodal distribution (typically produced by noise only samples), in which both algorithms underperformed. In this case, these purely autonomous algorithms (from the GTT class) do not meet the required standard. Nevertheless, the other examined GTTs were seen to maintain a low $P_{F A}$ as required.

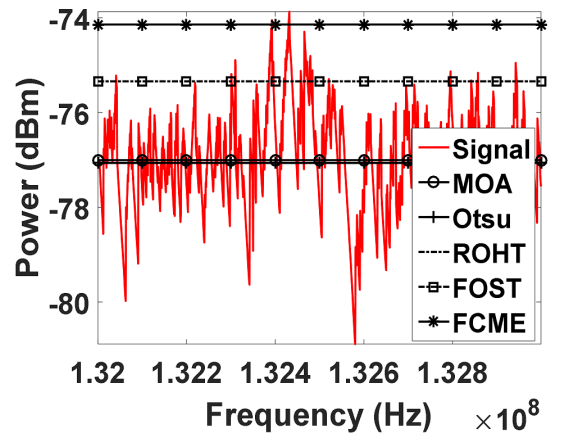

(a)

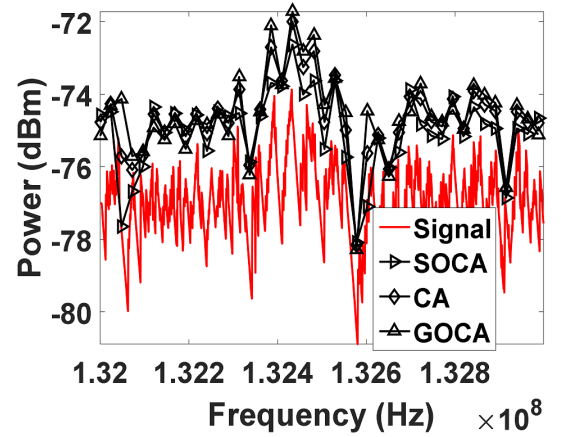

(b)

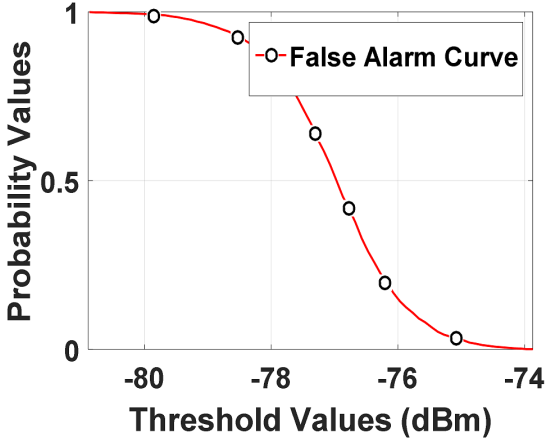

(c)

Fig. 5: (a) A real noise spectrum (132 - $133 \mathrm{MHz}$ ) being thresholded via different GTTs. (b) Thresholding via representative LTTs. (c) The computed probability of detection and the probability of false alarm for the dataset in (a) and (b)

4.2.2 Crowded FM Band $(88-108 \mathrm{MHz})$ : Signals acquired from the FM band were examined for the case of a highly crowded spectra. Essentially, this provided a scenario wherein the effect of several closely spaced signals on the different algorithms were studied. The threshold outcomes for the GTT and the LTT class are depicted in Fig. 6a and 6b, respectively. Likewise, the ROC curve is shown in Fig. 6c. In this case, we easily observe the presence of both the probability of detection, $P_{D}$ and probability of false alarm $P_{F A}$, curves, respectively. A summary of the point performance for each algorithm is provided in Table IV. It is seen that the LTT class experienced a grossly low detection rate. Conversely, the GTT class provided a better detection performance than the LTT class, particularly for the MOA, the Otsu and the FCME algorithms. The ROHT and the FOST on the other hand grossly under performed in the detection case. For the LTT class, we may explain that the closely spaced signals biased the threshold to higher values thus resulting in a gross signal masking effect. The ROHT and FOST 
TABLE IV: Detection and False Alarm Rate Performance for the different ATTs for the Crowded FM Band dataset (True Threshold $=-52 \mathrm{dBm}$

\begin{tabular}{ccccc}
\hline Class of ATT & Algorithms & Threshold (dBm) & $P_{D}(\%)$ & $P_{F A}(\%)$ \\
\hline \multirow{6}{*}{ GTTs } & MOA [46] & -53.05 & 100 & 5 \\
& Otsu [6] & -54.04 & 100 & 10 \\
& ROHT [6] & -39.33 & 12 & 0 \\
& FOST [34] & -40.98 & 18 & 0 \\
& FCME [7] & -55.57 & 100 & 19 \\
\hline \multirow{3}{*}{ LTTs } & CA-CFAR [16] & - & 26 & 4 \\
& SOCA-CFAR [48] & - & 58 & 28 \\
& GOCA-CFAR [17] & - & 11 & 4 \\
\hline
\end{tabular}

(in the GTT class) also experienced a strong large bias in the computation of the mean and standard deviation of the dataset, thus increasing the threshold value. This may be a drawback for both methods as noted in [46]. The Otsu and the MOA both performed well in both detection and false alarm terms. This is attributed to the presence of a bimodal distribution in the dataset resulting in better threshold computations. Summarily, the GTT class performed better than the LTT class considering a crowded FM band.

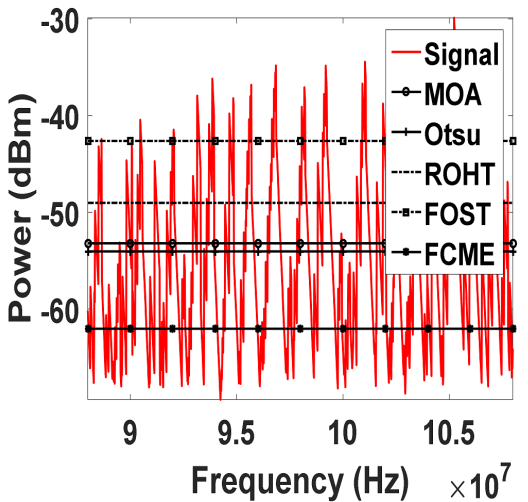

(a)

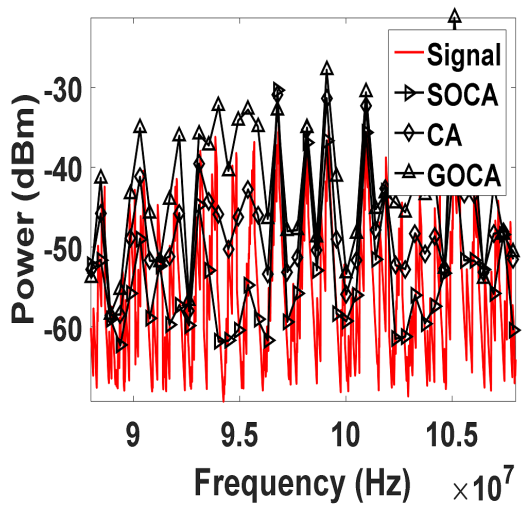

(b)

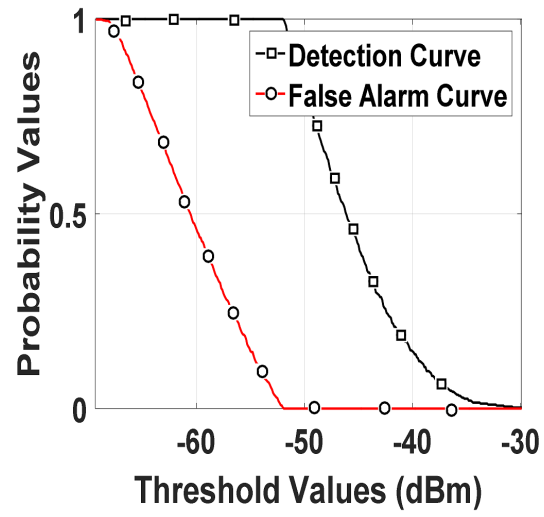

(c)

Fig. 6: (a) A crowded FM signal spectrum (88 - $108 \mathrm{MHz}$ ) being thresholded via different GTTs. (b) Thresholding via representative LTTs. (c) The computed probability of detection and the probability of false alarm for the dataset in (a) and (b)

4.2.3 Digital TV signals (720 - $732 \mathrm{MHz}$ ): We examined the case for Digital TV signals. The threshold values computed by the different GTT methods is shown in Fig. 7a, while Fig. 7b depicts for the LTT class. The ROC curves for this dataset shown in Fig. 7c indicates a large separation between the detection and false alarm curves. This phenomenon easily confirms the presence of a high Signal to Noise Ratio (SNR) condition in the dataset (easily verifiable from Fig. 7a). The point performance of each algorithm is provided in Table V. In this experiment, the LTT class achieved a grossly low detection performance at the expense of a low false alarm rate. The noise-like characteristics exhibited at the crest of the OFDM spectra may have biased the LTT methods into maintaining a constant false alarm rate. While the edges of the OFDM signal (obvious for the DTV band) caused a rise in the threshold values of the respective LTT methods. On the other hand, the GTT methods produced an appreciable level of detection and false rate performance except for the ROHT and the FOST algorithms. The ROHT and FOST suffered from a mean and standard deviation bias caused by the high powered OFDM signals varying rapidly at the crest of the OFDM spectra. Furthermore, the narrowband signal of the DTV band had little effect on the GTT methods, particularly for the MOA, the Otsu and the FCME algorithms. This can be considered an advantage of the GTT class. We see in this experiment that the GTT methods provided a better overall performance than the LTTs.

4.2.4 Analog TV signals (174 - $181 \mathrm{MHz}$ ): An Analog TV (ATV) band was examined capturing a transmitting station swept between $174-181 \mathrm{MHz}$. The video and audio peaks are easily discernible in Fig. 8a, while the valley between both peaks presents a noteworthy case to be examined. The LTT methods maintained a low false alarm rate at the expense of a low detection rate (see Table VI). The FCME algorithm from the GTT class produced a high detection rate albeit a slightly high corresponding false alarm rate. This high false alarm rate obviously violates the IEEE 802.22 standard [47]. The GTT methods also suffered a correspondingly low detection performance at the expense of low false alarm rates. Here, we draw attention to the valley between the video and audio peaks of the ATV spectra. The low power values in the valley strongly 


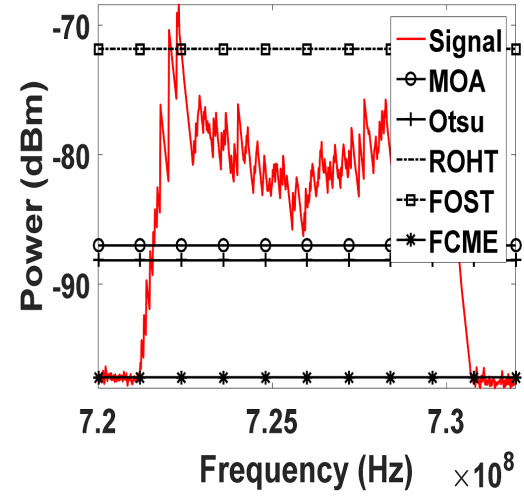

(a)

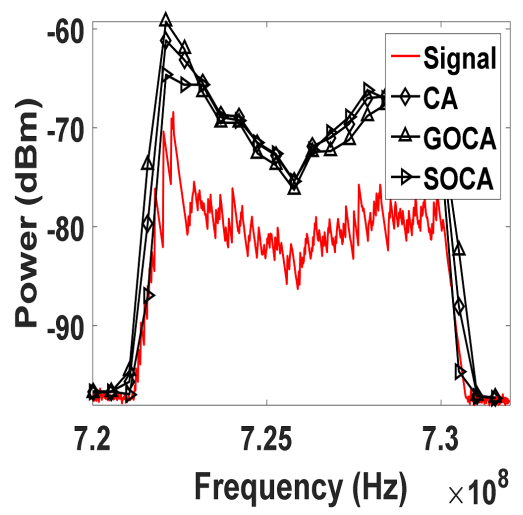

(b)

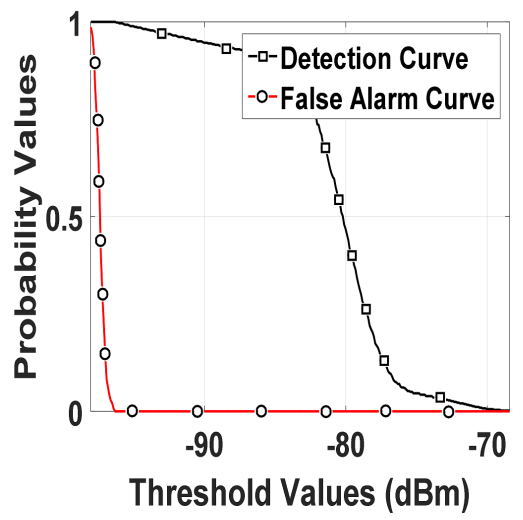

(c)

Fig. 7: (a) A DTV signal spectrum (720 - $732 \mathrm{MHz}$ ) being thresholded via different GTTs. (b) Thresholding via representative LTTs. (c) The computed probability of detection and the probability of false alarm for the dataset in (a) and (b)

TABLE V: Detection and False Alarm Rate Performance for the different ATTs for the Digital TV dataset (True Threshold $=-96.3 \mathrm{dBm}$

\begin{tabular}{ccccc}
\hline Class of ATT & Algorithms & Threshold $(\mathbf{d B m})$ & $P_{D}(\%)$ & $P_{F A}(\%)$ \\
\hline \multirow{3}{*}{ GTTs } & MOA [46] & -87.02 & 93 & 0 \\
& Otsu [6] & -88.17 & 93 & 0 \\
& ROHT [6] & -71.83 & 2 & 0 \\
& FOST [34] & -71.83 & 2 & 0 \\
& FCME [7] & -97.20 & 100 & 30 \\
\hline \multirow{3}{*}{ LTTs } & CA-CFAR [16] & - & 0 & 10 \\
& SOCA-CFAR [48] & - & 8 & 16 \\
& GOCA-CFAR [17] & - & 0 & 9 \\
\hline
\end{tabular}

affected the detection performance of the respective algorithms by causing an increase in their respective estimated threshold values. We consider this a limitation of the ATV technology known for its poor spectra utilization ratio as compared to its DTV counterpart. Thus, except for the FCME algorithm, the ATV experiments revealed both low detection and false alarm rates for both classes.

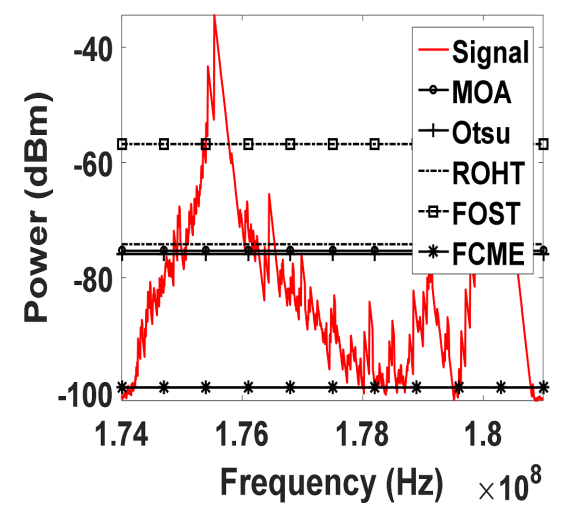

(a)

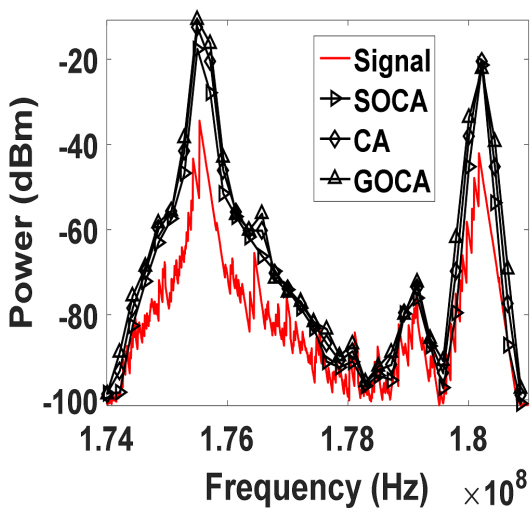

(b)

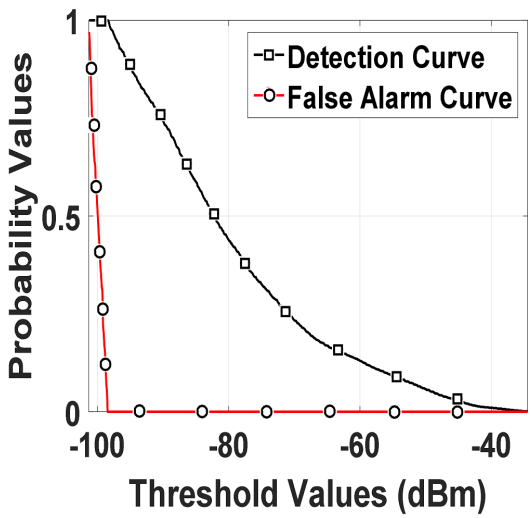

(c)

Fig. 8: (a) An ATV signal spectrum (174 - $181 \mathrm{MHz}$ ) being thresholded via different GTTs. (b) Thresholding via representative LTTs. (c) The computed probability of detection and the probability of false alarm for the dataset in (a) and (b)

4.2.5 Single FM Radio Station Signal $(90.2$ - 90.3 MHz): The spectra of a single FM radio station was acquired to examine the effect of narrow bandwidth on the performance of the both classes. Clear side lobes are noticeable in the measured band resulting in an increase the occupancy rate. From the ROC curves (see Fig. 9c), we notice a sudden and fast rise in the detection curve indicating a low noise level in the band. This implies that the sensed band belongs to a narrow spectra under a high occupancy rate. The results in Table VII reveal the poor detection performance of the LTT class. The ROHT and FOST 
TABLE VI: Detection and False Alarm Rate Performance for the different ATTs for the Analog TV dataset (True Threshold $=-98 \mathrm{dBm}$

\begin{tabular}{ccccc}
\hline Class of ATT & Algorithms & Threshold (dBm) & $P_{D}(\%)$ & $P_{F A}(\%)$ \\
\hline \multirow{6}{*}{ GTTs } & MOA [46] & -75.34 & 33 & 0 \\
& Otsu [6] & -75.90 & 34 & 0 \\
& ROHT [6] & -74.20 & 31 & 0 \\
& FOST [34] & -56.81 & 10 & 0 \\
& FCME [7] & -99.06 & 100 & 22 \\
\hline \multirow{3}{*}{ LTTs } & CA-CFAR [16] & - & 3 & 0 \\
& SOCA-CFAR [48] & - & 13 & 19 \\
& GOCA-CFAR [17] & - & 2 & 0 \\
\hline \multirow{6}{*}{} & & & &
\end{tabular}

TABLE VII: Detection and False Alarm Rate Performance for the different ATTs for the Single FM Radio Station dataset (True Threshold $=-65$ dBm)

\begin{tabular}{ccccc}
\hline Class of ATT & Algorithms & Threshold (dBm) & $P_{D}(\%)$ & $P_{F A}(\%)$ \\
\hline \multirow{6}{*}{ GTTs } & MOA [46] & -53.86 & 39 & 0 \\
& Otsu [6] & -55.01 & 45 & 0 \\
& ROHT [6] & -49.40 & 19 & 0 \\
& FOST [34] & -45.41 & 12 & 0 \\
& FCME [7] & -65.75 & 100 & 58 \\
\hline \multirow{3}{*}{ LTTs } & CA-CFAR [16] & - & 0 & 0 \\
& SOCA-CFAR [48] & - & 18 & 40 \\
& GOCA-CFAR [17] & - & 0 & 3 \\
\hline
\end{tabular}

algorithms of the GTT class performed poorly for reasons stated in Section 4.2.4. The FCME algorithm produced the highest detection performance at the expense of a high false alarm rate. The MOA and the Otsu were also below the detection range stipulated in the IEEE 802.22 specifications [47]. The presence of side lobes in the spectra and the deep valleys caused the general poor detection performance of the both classes in this experiment.

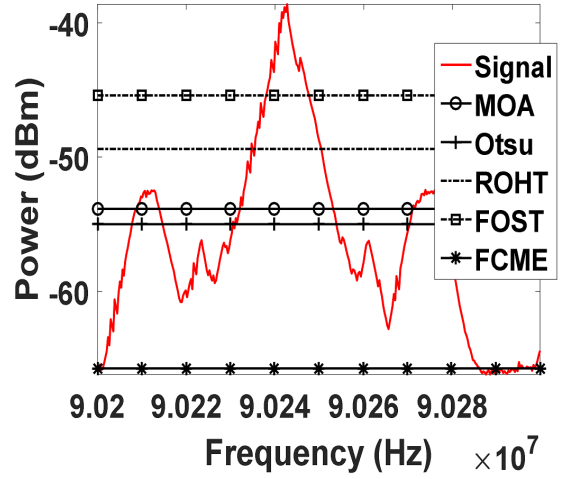

(a)

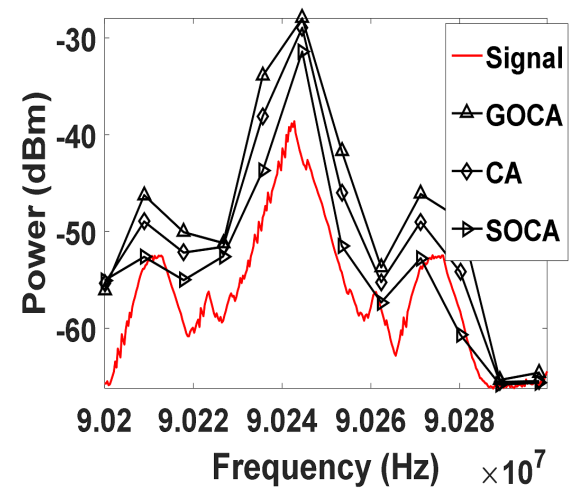

(b)

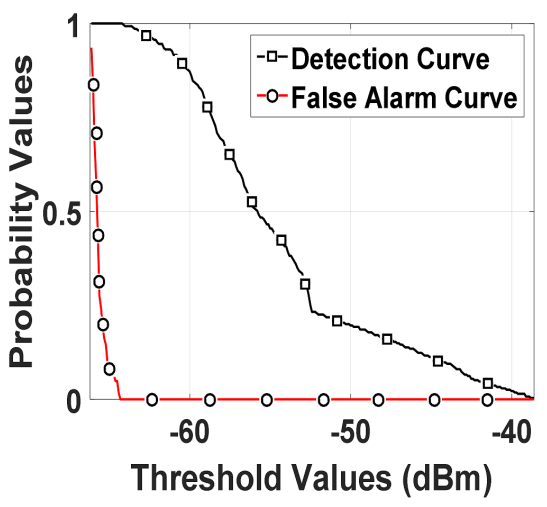

(c)

Fig. 9: (a) A Signal FM radio station band (90.2 - 90.3 MHz) being thresholded via different GTTs. (b) Thresholding via representative LTTs. (c) The computed probability of detection and the probability of false alarm for the dataset in (a) and (b)

4.2.6 Two Closely Spaced Signals (90 - 94 MHz): It often possible that high powered signals may bias the estimated threshold towards higher values that may mask low powered signals. This case was considered and investigated by evaluating the different algorithms over a spectrum containing two closely spaced signals with about $20 \mathrm{~dB}$ difference in their dynamic ranges. The estimated thresholds for the GTT class are shown in Fig. 10a, while that for the LTT class are shown in Fig. 10b. The ROC curve is shown in Fig. 10c. We notice a case for colored noise samples in the spectrum with a low noise level that slowly ramps upward. The GTT methods provided better detection performance than the LTT methods. The FCME algorithm performed well in this case, followed by the ROHT. The MOA, the Otsu, and the FOST algorithms fell below the detection standard specified by the IEEE 802.22 draft. The LTT methods missed both signals resulting in a $0 \%$ detection rate. Again, the GTT methods performed better than the LTT class in detection terms.

4.2.7 A Wide Sparse Spectrum with a Single Signal Source (424 - $454 \mathrm{MHz}$ ): We considered the case for wide band sensing with a single signal source in the band. This spectra is shown along with the threshold values for the GTT class in Fig. 11a, 


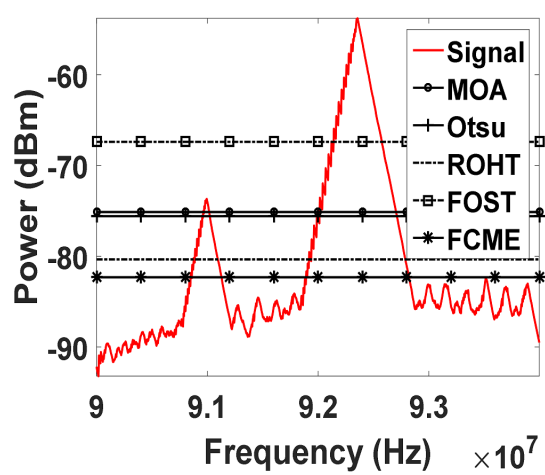

(a)

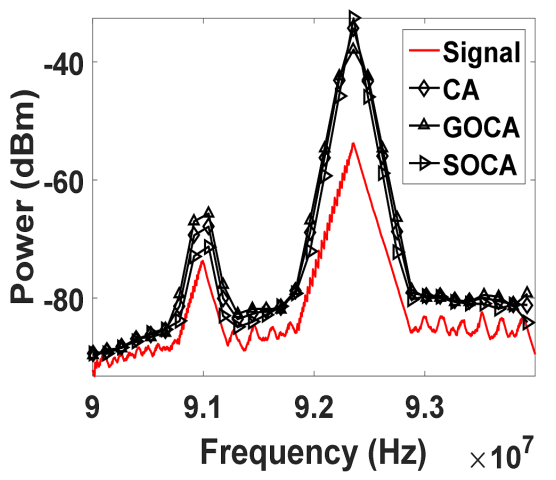

(b)

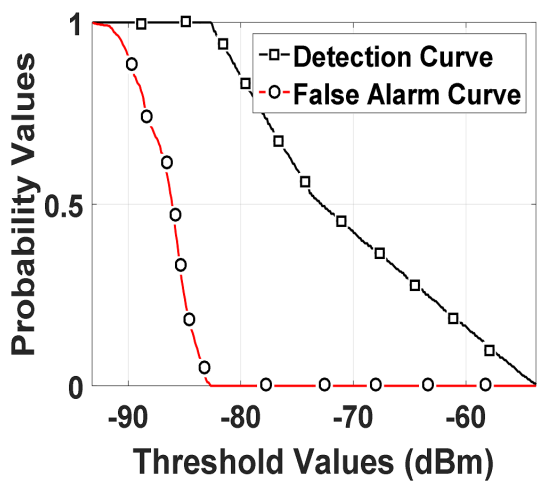

(c)

Fig. 10: (a) Spectrum of two different signals with wide difference in their Dynamic Range (90 - 94 MHz) being thresholded via different GTTs. (b) Thresholding via representative LTTs. (c) The computed probability of detection and the probability of false alarm for the dataset in (a) and (b)

TABLE VIII: Detection and False Alarm Rate Performance for the different ATTs for Two closely spaced signals $($ True Threshold $=-83$ dBm)

\begin{tabular}{ccccc}
\hline Class of ATT & Algorithms & Threshold $\mathbf{( d B m )}$ & $P_{D}(\%)$ & $P_{F A}(\%)$ \\
\hline \multirow{3}{*}{ GTTs } & MOA [46] & -75.13 & 60 & 0 \\
& Otsu [6] & -75.59 & 63 & 0 \\
& ROHT [6] & -80.35 & 87 & 0 \\
& FOST [34] & -67.39 & 36 & 0 \\
& FCME [7] & -82.32 & 98 & 0 \\
\hline \multirow{3}{*}{ LTTs } & CA-CFAR [16] & - & 0 & 0 \\
& SOCA-CFAR [48] & - & 0 & 2 \\
& GOCA-CFAR [17] & - & 0 & 0 \\
\hline
\end{tabular}

and for the LTT class in Fig. 11b. We notice from the ROC curves in Fig. 11c that the false alarm curve begins to rise at the lower end of the scale indicating a low noise level in the spectra. The point performances of the different algorithms are presented in Table IX. A poor detection performance is observed for the LTT methods at a low false alarm rate. We observe a considerable higher detection performance for the GTT methods. The FCME algorithm provided a remarkably good detection and false alarm performance as compared to the other GTTs. We notice a higher detection performance for the MOA and the Otsu as compared to the preceding experiments. The GTT methods are observed to have performed better than the LTT methods.

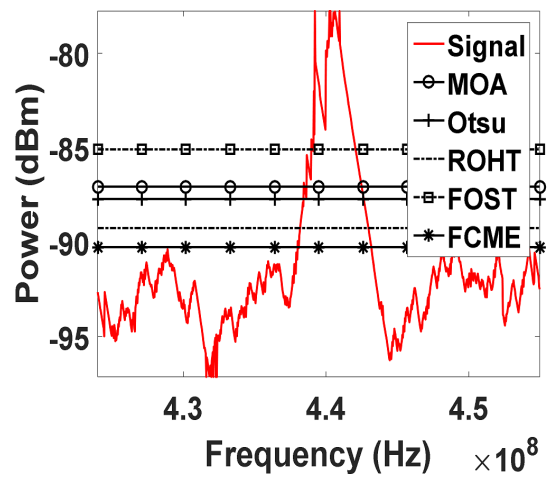

(a)

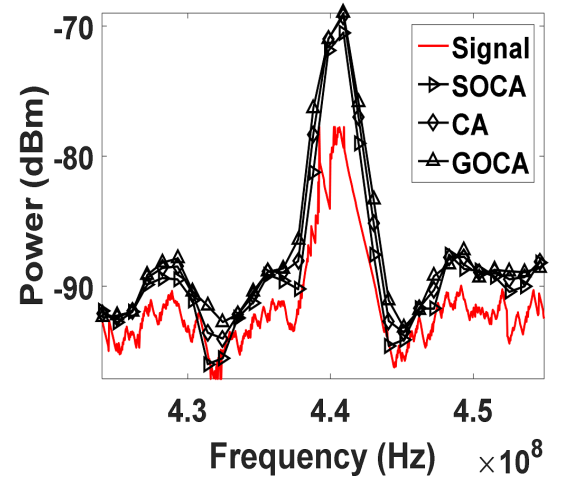

(b)

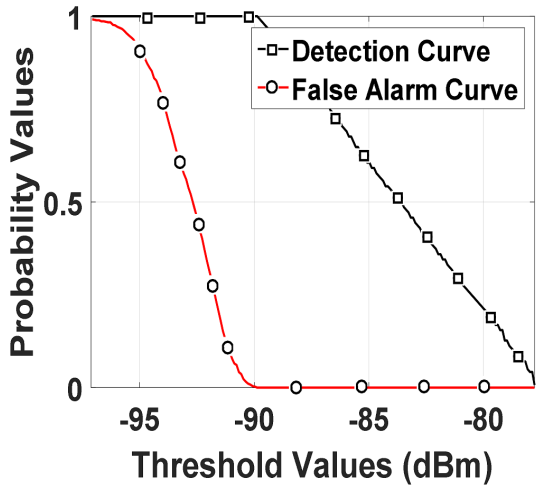

(c)

Fig. 11: (a) A wide sparse spectrum with a single signal source (424 - $454 \mathrm{MHz})$ being thresholded via different GTTs. (b) Thresholding via representative LTTs. (c) The computed probability of detection and the probability of false alarm for the dataset in (a) and (b)

\subsection{Discussion on the Performance of LTTs}

It is obvious that the representative LTTs considered in our study performed poorly in detection terms. We provide some reasons for this poor performance leveraging from the established theory in CFAR detection. First, we note that the noise 
TABLE IX: Detection and False Alarm Rate Performance for the different ATTs for the Sparse Spectrum with a single signal source $($ True Threshold $=-92$ $\mathrm{dBm}$

\begin{tabular}{ccccc}
\hline Class of ATT & Algorithms & Threshold (dBm) & $P_{D}(\%)$ & $P_{F A}(\%)$ \\
\hline \multirow{6}{*}{ GTTs } & MOA [46] & -87.06 & 79 & 0 \\
& Otsu [6] & -87.71 & 85 & 0 \\
& ROHT [6] & -89.25 & 96 & 0 \\
& FOST [34] & -85.09 & 62 & 0 \\
& FCME [7] & -90.26 & 100 & 1 \\
\hline \multirow{3}{*}{ LTTs } & CA-CFAR [16] & - & 0 & 0 \\
& SOCA-CFAR [48] & - & 1 & 6 \\
& GOCA-CFAR [17] & - & 0 & 0 \\
\hline
\end{tabular}

samples considered in this paper, which are commonly found in most communication systems, exhibit notable homogenous patterns typically produced by exponentially controlled distributions. Secondly, the signal samples found in our study were typically closely spaced, averagely high powered, and distributed over a large contiguous range of frequencies (broadband). These are well known conditions under which CFAR techniques (LTTs) are theoretically known to perform poorly. In particular, Gandhi et al., in [12, pp.431] noted that it is impossible under these conditions for a CFAR technique to detect contiguous and independent targets (signal samples) of identical strength. This is because threshold factors employed by all LTTs become biased towards higher values by the presence of several high powered signals in the reference window. Furthermore, the use of a small finite window size and a predefined false alarm rate typically results in large detection losses. These are a few theoretical reasons responsible for the poor performance of LTTs, for which the empirical results of our study now further support.

\section{CONCLUSION}

In this paper, we have conducted a systematic comparative analysis of local and global thresholding techniques (LTTs and GTTs) for energy detection in Cognitive Radio (CR). This study was considered necessary in order to establish a sum-up synopsis regarding the effective performance range and the desirable operating conditions under which both classes best apply in CR. Our findings suggest that GTTs generally perform better than their LTT counterparts particularly in detection terms. This poor detection performance exhibited by LTTs may be linked to the threshold biasing effect originating from the presence of contiguous signal samples in the algorithm's computing window set. On the other hand, GTTs were found to be more suited for CR applications with the FCME algorithm particularly noted for its better performance than the other GTTs considered in this paper. The results of this paper will be beneficial to CR developers mainly because it provides comparative evidences suggesting the need for new improved ATTs belonging to the GTT class rather than the LTT class.

\section{REFERENCES}

[1] T. Yucek and H. Arslan, "A survey of spectrum sensing algorithms for cognitive radio applications," IEEE communications surveys \& tutorials, vol. 11, no. 1, pp. 116-130, 2009.

[2] T. M. Chiwewe and G. P. Hancke, "Fast convergence cooperative dynamic spectrum access for cognitive radio networks," IEEE Transactions on Industrial Informatics, vol. PP, pp. 1-1, Dec. 2017.

[3] J. Mitola and G. Q. Maguire, "Cognitive radio: Making software radios more personal," IEEE personal communications, vol. 6, no. 4, pp. 13-18, 1999.

[4] S. S. Oyewobi and G. P. Hancke, "A survey of cognitive radio handoff schemes, challenges and issues for industrial wireless sensor networks (CR-IWSN)," Journal of Network and Computer Applications, vol. 97, pp. 140-156, Nov. 2017.

[5] R. Tandra and A. Sahai, "Fundamental limits on detection in low SNR under noise uncertainty," in IEEE International Conference on Wireless Networks, Communications and Mobile Computing, vol. 1, pp. 464-469, 2005.

[6] D. Datla, A. M. Wyglinski, and G. Minden, "A spectrum surveying framework for dynamic spectrum access networks," IEEE Transactions on Vehicular Technology, vol. 58, no. 8, pp. 4158-4168, 2009.

[7] J. J. Lehtomki, J. Vartiainen, M. Juntti, and H. Saarnisaari, "Spectrum sensing with forward methods," in IEEE conference on Military communications, pp. 2217-2223, IEEE, Oct. 2006.

[8] E. U. Ogbodo, D. Dorrell, and A. M. Abu-Mahfouz, "Cognitive Radio Based Sensor Network in Smart Grid: Architectures, Applications and Communication Technologies," IEEE Access, vol. 5, no. c, pp. 19084-19098, 2017.

[9] M. Ndiaye, G. P. Hancke, and A. M. Abu-Mahfouz, "Software defined networking for improved wireless sensor network management: A survey," Sensors, vol. 17, no. 5, p. 1031, 2017.

[10] H. I. Kobo, A. M. Abu-Mahfouz, and G. P. Hancke, "A survey on software-defined wireless sensor networks: Challenges and design requirements," IEEE Access, vol. 5, pp. 1872-1899, 2017.

[11] J. Wan, S. Tang, D. Li, S. Wang, C. Liu, H. Abbas, and A. V. Vasilakos, "A manufacturing big data solution for active preventive maintenance," IEEE Transactions on Industrial Informatics, vol. 13, pp. 2039-2047, Aug. 2017.

[12] P. P. Gandhi and S. A. Kassam, "Analysis of CFAR processors in homogeneous background," IEEE Transactions on Aerospace and Electronic systems, vol. 24 , no. 4, pp. 427-445, 1988.

[13] J. J. Lehtomaki, M. Juntti, and H. Saarnisaari, “CFAR strategies for channelized radiometer," IEEE signal processing letters, vol. 12, pp. 13-16, Jan. 2005.

[14] J. J. Lehtomaki, J. Vartiainen, M. Juntti, and H. Saarnisaari, "CFAR outlier detection with forward methods," IEEE Transactions on Signal Processing, vol. 55, no. 9, pp. 4702-4706, 2007.

[15] A. J. Onumanyi, "Spectral data for evaluating and validating threshold estimators in cognitive radio [data set]," Mendeley, Jan. 2018. 
[16] H. Finn and R. Johnson, "Adaptive detection mode with threshold control as a function of spatially sampled clutter- level estimates (adaptive detection mode for surveillance radar, using detection threshold proportional to spatially sampled clutter level estimates for regulation of false alarm probability)," RCA review, vol. 29, pp. 414-464, 1968.

[17] V. G. Hansen, "Constant false alarm rate processing in search radars (receiver output noise control)," Radar- Present and future, pp. 325-332, 1973.

[18] G. V. Trunk, "Range resolution of targets using automatic detectors," IEEE Transactions on Aerospace and Electronic Systems, no. 5, pp. 750-755, 1978.

[19] H. M. Finn, "A CFAR design for a window spanning two clutter fields," IEEE Transactions on Aerospace and Electronic Systems, no. 2, pp. 155-169, 1986.

[20] H. Rohling, "New CFAR-processor based on an ordered statistic," in International Radar Conference, pp. 271-275, 1985.

[21] S. D. Himonas and M. Barkat, "Automatic censored CFAR detection for nonhomogeneous environments," IEEE Transactions on Aerospace and Electronic systems, vol. 28, no. 1, pp. 286-304, 1992.

[22] M. E. Smith and P. K. Varshney, "Intelligent CFAR processor based on data variability," IEEE Transactions on Aerospace and Electronic Systems, vol. 36, no. 3, pp. 837-847, 2000 .

[23] A. Zaimbashi, M. R. Taban, and M. M. Nayebi, "Distributed fuzzy CFAR detection for weibull clutter," IEICE transactions on communications, vol. 91, no. 2, pp. 543-552, 2008.

[24] A. Zaimbashi, "An adaptive cell averaging-based CFAR detector for interfering targets and clutter-edge situations," Digital Signal Processing, vol. 31, pp. 59-68, 2014.

[25] J. J. Lehtomaki, R. Vuohtoniemi, and K. Umebayashi, "On the measurement of duty cycle and channel occupancy rate," IEEE Journal on Selected Areas in Communications, vol. 31, pp. 2555-2565, Nov. 2013.

[26] D. Malafaia, J. Vieira, and A. Tom, "Adaptive threshold spectrum sensing based on expectation maximization algorithm," Physical Communication, vol. 21, pp. 60-69, 2016.

[27] K. Berbra, M. Barkat, F. Gini, M. Greco, and P. Stinco, "A fast spectrum sensing for CP-OFDM cognitive radio based on adaptive thresholding," Signal Processing, vol. 128, pp. 252-261, 2016.

[28] D. R. Joshi, D. C. Popescu, and O. A. Dobre, "Adaptive spectrum sensing with noise variance estimation for dynamic cognitive radio systems," in In 44th IEEE Annual Conference on Information Sciences and Systems (CISS), 2010, pp. 1-5, 2010.

[29] D. M. M. Plata and A. G. A. Reatiga, "Evaluation of energy detection for spectrum sensing based on the dynamic selection of detection-threshold," Procedia Engineering, vol. 35, pp. 135-143, 2012.

[30] N. Wang, Y. Gao, and L. Cuthbert, "Spectrum sensing using adaptive threshold based energy detection for OFDM signals," in IEEE International Conference on Communication Systems (ICCS), pp. 359-363, 2014.

[31] D. Teo, K. Zhong, and B. C. Ng, "An iterative threshold selection algorithm for cooperative sensing in a cognitive radio network," in IEEE Symposium on New Frontiers in Dynamic Spectrum, pp. 1-8, Apr. 2010.

[32] S. K. Srivastava and A. Banerjee, "n-ratio logic based cooperative spectrum sensing using double threshold energy detection," in 4th IEEE International Conference on Cognitive Radio Oriented Wireless Networks and Communications, pp. 1-6, 2009.

[33] D. Datla, A. M. Wyglinski, and G. J. Minden, "A statistical approach to spectrum measurement processing," Information and TelecommunicationsTechnology, 2007.

[34] A. Gorcin, K. A. Qaraqe, H. Celebi, and H. Arslan, "An adaptive threshold method for spectrum sensing in multi-channel cognitive radio networks," in 17th IEEE International Conference on Telecommunications (ICT), pp. 425-429, IEEE, 2010.

[35] X. Ling, B. Wu, H. Wen, P.-H. Ho, Z. Bao, and L. Pan, "Adaptive threshold control for energy detection based spectrum sensing in cognitive radios," IEEE Wireless Communications Letters, vol. 1, no. 5, pp. 448-451, 2012.

[36] R. K. Dubey and G. Verma, "Improved spectrum sensing for cognitive radio based on adaptive double threshold," International Journal of Emerging Trends in Electrical and Electronics (IJETEE), vol. 11, no. 2, pp. 1-6, 2015.

[37] J. Avila and K. Thenmozhi, "Adaptive double threshold with multiple energy detection technique in cognitive radio," Research Journal of Applied Sciences, Engineering and Technology, vol. 10, no. 11, pp. 1336-1342, 2015.

[38] B. Ahuja, K. Sharma, M. Abo-Zahhad, O. Amin, M. Farrag, A. Ali, and A. Rachman, "Adaptive double threshold based spectrum sensing for cognitive radio networks," International Journal of Energy, Information and Communications, vol. 5, no. 6, pp. 1-16, 2014.

[39] A. Bagwari and G. S. Tomar, "Two-stage detectors with multiple energy detectors and adaptive double threshold in cognitive radio networks," International Journal of Distributed Sensor Networks, vol. 2013, 2013.

[40] J. Xie and J. Chen, "An adaptive double-threshold spectrum sensing algorithm under noise uncertainty," in 12th IEEE International Conference on Computer and Information Technology (CIT), pp. 824-827, 2012.

[41] S. Q. Liu, B. J. Hu, and X. Y. Wang, "Hierarchical cooperative spectrum sensing based on double thresholds energy detection," IEEE Communications Letters, vol. 16, no. 7, pp. 1096-1099, 2012.

[42] D. M. Martnez and A. G. Andrade, "Adaptive energy detector for spectrum sensing in cognitive radio networks," Computers \& Electrical Engineering, 2015.

[43] S. Liu, J. Shen, R. Zhang, Z. Zhang, and Y. Liu, "Information theoretic criterion-based spectrum sensing for cognitive radio," IET communications, vol. 2, no. 6, pp. 753-762, 2008.

[44] F. Salahdine, N. Kaabouch, and H. El Ghazi, "A survey on compressive sensing techniques for cognitive radio networks," Physical Communication, vol. 20, pp. 61-73, 2016.

[45] N. Otsu, "A threshold selection method from gray-level histograms," Automatica, vol. 11, no. 285-296, pp. 23-27, 1975.

[46] A. J. Onumanyi, E. N. Onwuka, A. M. Aibinu, O. C. Ugweje, and M. J. Salami, "A modified Otsu's algorithm for improving the performance of the energy detector in cognitive radio," AEU - International Journal of Electronics and Communications, vol. 79, pp. 53-63, 2017.

[47] IEEE802.22, "Enabling broadband wireless access using cognitive radio technology and spectrum sharing in white spaces," IEEE 802.22 Working Group on Wireless Regional Area Networks, 2011.

[48] M. Weiss, "Analysis of some modified cell-averaging CFAR processors in multiple-target situations," IEEE Transactions on Aerospace and Electronic Systems, no. 1, pp. 102-114, 1982.

[49] T. Fawcett, "An introduction to ROC analysis," Pattern recognition letters, vol. 27, no. 8, pp. 861-874, 2006.

[50] J. J. Lehtomaki, J. Vartiainen, and H. Saarnisaari, "Adaptive FCME-based threshold setting for energy detectors," No. 33, pp. 1 - 5, Oct. 2011.

[51] J. Lehtomäki, S. Salmenkaita, J. Vartiainen, J. P. Mäkelä, R. Vuohtoniemi, and M. Juntti, "Measurement studies of a spectrum sensing algorithm based on double thresholding," in 2009 2nd International Workshop on Cognitive Radio and Advanced Spectrum Management, CogART 2009, pp. 69-73, IEEE, may 2009. 